\title{
Role of CD97 isoforms in gastric carcinoma
}

\author{
DAREN LIU ${ }^{1,2}$, BOGUSZ TROJANOWICZ $^{2}$, YVONNE RADESTOCK ${ }^{2}$, TING FU $^{2}$, \\ KATHRIN HAMMJE ${ }^{2}$, LI CHEN $^{1}$ and CUONG HOANG-VU ${ }^{2}$
}

\author{
${ }^{1}$ Department of Surgery, Second Affiliated Hospital, College of Medicine, Zhejiang University, Hangzhou, P.R. China; \\ ${ }^{2}$ Clinics of General, Visceral and Vascular Surgery, Martin Luther University, Halle/Saale, Germany
}

Received January 15, 2010; Accepted March 5, 2010

\section{DOI: 10.3892/ijo_00000625}

\begin{abstract}
The aim of this study was to elucidate the role of CD97 isoforms in gastric carcinoma. Out of four gastric cancer cell lines investigated, BGC-823 cells demonstrating low CD97 protein expression were stably transfected with pcDNA3.1 vector containing CD97/EGF1,2,5 or CD97/EGF1,2,3,4,5 inserts. Behavior of transfected cells was systematically investigated by employing proliferation, motility and invasive assays. As a result, we found that over-expression of CD97/ EGF1,2,5 isoform correlated with increased motile and invasive ability of the clones. Furthermore, CD97/EGF1,2,5 isoform over-expression (3.8 times higher) was followed by significant decrease of CD97/EGF1,2,3,4,5 isoform (10.3 times lower). In contrast, CD97/EGF1,2,3,4,5 clones revealed significantly reduced invasive properties as compared with corresponding controls. The changes in acetylation status were one of the possible mechanisms affecting behavior of transfected cells. We concluded from the study that CD97 is closely related with advanced stages and higher invasiveness of gastric carcinoma. The study further lightened the tumor promoting role of CD97 small isoform in cancer progression and indicated the possible suppressive properties of the full length isoform of CD97.
\end{abstract}

\section{Introduction}

CD97 is a member of epidermal growth factor (EGF) transmembrane seven (TM7) superfamily, which is a group of adhesion class seven-span transmembrane receptors originally found to be expressed by hematopoietic cells $(1,2)$. CD97 is composed of 2 noncovalently associated subunits (3): the $\alpha$-subunit comprises the majority of the extracellular domain and contains the adhesion motifs, while the $\beta$-subunit comprises the 7 TM receptor with a short extracellular extension. CD97 is produced in alternatively spliced forms that contain three $(\mathrm{EGF1}, 2,5)$, four (EGF1,2,3,5), or five (EGF1,2,3,4,5)

Correspondence to: Dr Cuong Hoang-Vu, Clinics of General, Visceral and Vascular Surgery, Martin Luther University, Magdeburger Strasse 18, D-06097 Halle (Saale), Germany

E-mail: hoang-vu@medizin.uni-halle.de

Key words: CD97, isoform, gastric carcinoma repeated EGF domains (4). According to different binding affinity, the smallest isoform which contains 3 EGF repeats (CD97/EGF1,2,5) interacts with CD55, also known as decayaccelerating factor (DAF) (5-7). The full length isoform (CD97/EGF1,2,3,4,5) binds exclusively glycosaminoglycan (GAG) chondroitin sulfate (CS) $(8,9)$. CD97 was abundantly detected in various normal tissues and advanced stages of thyroid, colorectal, gastric, pancreatic, esophageal and oral squamous cell carcinomas (10-14). In thyroid carcinomas, CD97 expression was found to correlate with the grade of differentiation and was exclusively expressed in highly dedifferentiated anaplastic carcinomas, while in welldifferentiated papillary or follicular carcinomas, CD97 expression was very low or negative (10). In gastric carcinoma tissues, the majority of gastric adenocarcinomas was CD97 positive (60-88\%) and mostly expressed at the invasion front, but negative or only slightly positive in normal gastric mucosa (15). Furthermore, various studies confirmed that elevated expression of CD97 in gastric cancer is associated with the dedifferentiation and aggressiveness of tumor cells and directly correlates with other clinicopathological parameters such as TNM classification (14). Recently, in colorectal cancer cells the interaction between the small isoform of CD97 (CD97/EGF1,2,5) and its ligand CD55 led to increased motility of the cells and elevated proteolytic activity of matrix metalloproteinases or chemokine secretion $(16,17)$. These observations raised several questions concerning the possible roles of CD97 isoforms in gastric carcinoma progression. We assume that increased motility of the cells and tumor progression occurs in isoform-specific manner. However, the specific role of the three CD97 isoforms, especially the small and full length isoforms, their mechanisms and interactions during those processes still need to be clarified.

The aim of this work was to determine the expression of CD97 in relation to the invasive abilities of gastric garcinoma cells, as well as to elucidate the role of CD97 isoforms in CD97/EGF1,2,5 and CD97/EGF1,2,3,4,5 stably transfected BGC-823 gastric cancer cells.

\section{Materials and methods}

Cell culture. Stomach adenocarcinoma cell line BGC-823 cells were propagated in Dulbecco's minimal essential medium (DMEM)/Ham's F12 (Biochrom, Berlin, Germany), MGC-801 and AGS cells were cultured in RPMI-1640 medium 
(Biochrom, Berlin, Germany), and HGC-27 cells were cultured in minimal essential medium (MEM, Biochrom). All media were supplemented with $10 \%$ fetal calf serum (FCS) and $1 \%$ penicillin/streptomycin (Biochrom AG). The cells were grown in standard humidified incubator in $5 \% \mathrm{CO}_{2}$ at $37^{\circ} \mathrm{C}$ and passaged every 4-7 days using trypsin-EDTA (Life Technologies, Karlsruhe, Germany).

Total RNA extraction and reverse transcriptase-polymerase chain reaction ( $R T-P C R)$. Total RNA from BGC-823wild-type cells and stable BGC-823-CD97 transfectants was extracted using TRIzol reagent according to manufacturer's instructions (Life Technologies). Total RNA ( $1 \mu \mathrm{g})$ was used for the first-strand cDNA synthesis using the Superscript reverse transcriptase kit and $500 \mathrm{ng} / \mathrm{ml}$ of oligo $\mathrm{d}(\mathrm{T})$ primers (Life Technologies). To exclude genomic amplification of prepro-CD97, specific intron-spanning oligonucleotide primers, which are suitable for amplification of all 3 CD97 isoforms (sense-actctgccgggagctgaaac; antisense-tggatggtgacctcggctga), were employed. RT-PCR reactions were performed in a $25-\mu 1$ solution containing $4 \mu \mathrm{l}$ of cDNA, $2.5 \mu \mathrm{l}$ of $10 \mathrm{X}$ Advantage 2 polymerase mix buffer, $10 \mathrm{nmol} / \mathrm{l}$ of $\mathrm{dNTP}, 20 \mathrm{pmol}$ of the primer, and $2 \mathrm{U}$ TaqDNA-polymerase (Life Technologies). PCR cycles consisted of an initial denaturation step for $5 \mathrm{~min}$ at $95^{\circ} \mathrm{C}$, followed by 35 cycles of denaturation at $94^{\circ} \mathrm{C}$ for $30 \mathrm{sec}$, annealing for $45 \mathrm{sec}$ at $61^{\circ} \mathrm{C}$, elongation for $45 \mathrm{sec}$ at $72^{\circ} \mathrm{C}$, and a final extension cycle for $5 \mathrm{~min}$ at $72^{\circ} \mathrm{C}$. PCR products were visualized on a $1 \%$ agarose gel containing $0.05 \%$ ethidium bromide. Semiquantitative RT-PCR analysis was performed with the Bio 1D software (LTF, Wasserburg, Germany) and $18 \mathrm{~S}$ served as normalizing marker. For sequence analysis, amplicons were purified by Magic column extraction, cloned into the pGEM-T vector (both Promega, Heidelberg, Germany), and sequenced in both directions using the PRISM dye Deoxy Terminator cycle sequencing kit (Perkin-Elmer, Foster City, CA) and T7 or SP6 sequencing primers.

Generation of stable CD97 transfectants. PcDNA3.1/Zeo ${ }^{+}$ vector containing CD97/EGF1,2,3,4,5 or CD97/EGF1,2,5 inserts and empty plasmid alone were transfected into BGC-823 cells using Lipofectamine 2000 according to manufacturer's protocol (Life Technologies). Selection of the clones was initiated 2 days after transfection by employing $100 \mu \mathrm{g} / \mathrm{ml}$ of zeocin (Life Technologies). Two weeks after transfection, positive clones were selected and maintained in fresh medium containing Zeocin at final concentration of $100 \mu \mathrm{g} / \mathrm{ml}$. Medium was changed every 2-3 days and transfectants were passaged every 5-6 days. Over-expression of CD97 was verified by RT-PCR and Western blot analysis.

Cell proliferation assay (MTT assay). BGC-823 wild-type cells, empty plasmid control and stable BGC-CD97 transfectants were plated in $200 \mu \mathrm{l}$ of DMEM with $10 \%$ FCS at $2.5 \times 10^{4}$ cells/well in 96-well plates. After overnight incubation, the growth medium was replaced with serum-free medium. At each time-point (24, 48 and $72 \mathrm{~h}), 20 \mu \mathrm{l}$ of $5 \mathrm{mg} / \mathrm{ml} \mathrm{MTT}$ (Sigma) was added to each well and incubated at $37^{\circ} \mathrm{C}$ for $4 \mathrm{~h}$ to allow the reduction of MTT to formazan. Formazan crystals were dissolved in $100 \mu \mathrm{l}$ DMSO and measured at $540 \mathrm{~nm}$ using ELISA reader (TECAN, Austria GmbH).
Soft agar colony formation. Two-layered soft agar assays were performed in 6-well plates. The bottom layer of agar $(1.5 \mathrm{ml}$ per well) consisted of $2.5 \mathrm{ml}$ of $3 \%$ agar (Roth, Karlsruhe, Germany) in sterile water, $1.5 \mathrm{ml}$ of FCS (Biochrom), $22.5 \mu 1$ of Zeocin (75 $\mu \mathrm{g} / \mathrm{ml}$, Life Technologies), $150 \mu 1$ of a 1:1,000 dilution of amphotericin B $(0.25 \mu \mathrm{g} / \mathrm{ml}$; Sigma), and $450 \mu 1$ of a 1:1,000 dilution of gentamicin (10 $\mu \mathrm{g} / \mathrm{ml}$; Sigma) added to $15 \mathrm{ml}$ with Ham's F12 medium. Once solidified at room temperature for $10 \mathrm{~min}, 20,000$ of BGC-CD97/EGF1,2,3,4,5 or BGC-CD97/EGF1,2,5 or BGC-empty plasmid clones were mixed into $1 \mathrm{ml}$ of upper agar layer prepared from a stock consisting of $0.8 \mathrm{ml}$ of $3 \%$ agar, $10 \%$ FCS, $11.25 \mu 1$ of Zeocin (75 $\mu \mathrm{g} / \mathrm{ml}), 75 \mu \mathrm{l}$ (1:1,000 dilution) of amphotericin B, $225 \mu \mathrm{l}$ (1:1,000 dilution) of gentamicin in $15 \mathrm{ml}$ of culture medium. This cell suspension was carefully layered on the top of the bottom layer. Once the top agar layer had solidified, $1 \mathrm{ml}$ of the culture medium was carefully added and changed once a week. After 4 weeks, cell colonies in the agar were stained overnight at $37^{\circ} \mathrm{C}$ in a $5 \% \mathrm{CO}_{2}$ atmosphere with $200 \mu \mathrm{l}$ of iodo-tetrazolium chloride $(5 \mathrm{mg} / \mathrm{ml}$; Sigma). Stained cell colonies were examined by light microscopy (Zeiss).

Migration and invasion assays. The migration and invasion assays were evaluated in 24-well Transwell chambers (Costar, Bodenheim, Germany). The upper and lower culture compartments were separated by polycarbonate filters with $8 \mu \mathrm{m}$ pore size. For invasion assay, the upper site of the filters (upper chamber) was coated with $1 \mathrm{mg} / \mathrm{ml}$ of gelatin (Autogen Bioclear, UK) before seeding the cells. BGC-CD97/ EGF1,2,3,4,5, BGC-CD97/EGF1,2,5 or BGC-empty plasmid clones were seeded at $2 \times 10^{4}$ cells/well in Ham's F12 medium without FCS in the upper chamber and the lower chamber was filled with medium with $10 \%$ FCS. After 24-h incubation in a $5 \% \mathrm{CO}_{2}$ atmosphere at $37^{\circ} \mathrm{C}$, cells remaining on top of the filter were wiped off with cotton swabs and those transfectants that had traversed the membrane pores to the lower surface of the membrane were washed with chilled PBS, incubated for $5 \mathrm{~min}$ in 1:1 PBS/methanol (Merck, Darmstadt, Germany) and 15 min in methanol before staining with $0.1 \%$ toluidine blue (Merck) in $2.5 \%$ sodium carbonate (Roth). Migrated cells were counted by light microscopy (Zeiss) in four separate high-power fields per filter. Migration assay employed the same two-chambered 24-well plate filter assay but the top of the filter was not pre-coated with gelatin. All experiments were performed at least in triplicates and were expressed as mean \pm SEM.

Western blot analysis. CD97 protein expression was analyzed by Western blot analysis. BGC-823 wild-type cells BGCCD97/EGF1,2,3,4,5, BGC-CD97/EGF1,2,5 or BGC-empty plasmid clones were seeded at $1.5 \times 10^{5} /$ well in $25 \mathrm{~cm}^{2}$ flasks and cultured in medium with $10 \%$ FCS until $70 \%$ of confluency. Total proteins were isolated with $2 \mathrm{X}$ extraction buffer (125 mM Tris-HCl pH 6.8; 4\% sodium dodecylsulfate (SDS); $20 \%$ glycerol; $10 \%$ mercaptoethanol (ME); $2 \%$ bromophenol blue) plus protease inhibitors cocktail (all reagents from Sigma). Proteins were resolved on a $12 \%$ SDS gel, blotted at $1 \mathrm{~mA} / \mathrm{gel} \mathrm{cm}^{2}$ for $2 \mathrm{~h}$ onto Hybond-ECL nitrocellulose membranes (Amersham, Freiburg, Germany). Membranes with blotted proteins were blocked for $2.5 \mathrm{~h}$ 
with 5\% milk in PBS plus 0.02\% Tween-20 (PBST; Sigma) and incubated overnight at $4^{\circ} \mathrm{C}$ with the mouse polyclonal CD97 antibody (1:5,000; Abnova). Secondary HRP-conjugated rabbit anti-mouse IgGs was used at 1:20,000 for $1 \mathrm{~h}$ at RT. Immunoreactive protein bands were visualized with the ECL kit (Amersham). The same membranes were reprobed with mAbs specific to human $\beta$-actin prior to incubation in stripping solution (2\% SDS; $125 \mathrm{mM}$ Tris- $\mathrm{HCl}, \mathrm{pH} 8.0 ; 0.7 \% \mathrm{ME}$ ) and the block buffer (5\% milk in PBS plus $0.02 \%$ Tween-20). $B$-actin was visualized with secondary goat anti-mouse antibodies (Sigma, 1:20,000 in blocking buffer for $1 \mathrm{~h}$ ) and ECL-kit (Amersham).

Immunohistochemistry and immunofluorescence. Dako REAL $^{\mathrm{TM}}$ Detection Systems (LSAB ${ }^{+}$) was used for immunohistochemistry. Wild-type cells and BGC-823 transfectants were plated on sterile glass slides at $1 \times 10^{5} / \mathrm{ml}$ and incubated in a humidified atmosphere at $5 \% \mathrm{CO}_{2}$ for 2 to 3 days before fixation with cold methanol. The cells were incubated overnight at $4^{\circ} \mathrm{C}$ with primary antibody to CD97 (1:200) in blocking solution (Dako). Biotinylated link universal (Dako), Streptaridin-HRP (Dako) and substrate (Dako) were added subsequently according to the protocol. Mayer's Hemalaun solution (Applichem) was used for the visualization of the nuclei.

For the immunofluorescence the cells were fixed with $4 \%$ formaldehyde/PBS solution. After permeabilization with $1 \%$ Triton, slides were incubated overnight at $4^{\circ} \mathrm{C}$ with primary CD97 antibody (1:200) in blocking solution (Dako). Parallel, overnight incubations $\left(4^{\circ} \mathrm{C}\right.$ in blocking solution) with antibodies raised against acetylated-tubulin (1:100; Molecular probes) and FITC-conjugated $\alpha$-tubulin (1:100; Dianova, Hamburg, Germany) were employed for double detection of both tubulins. After washing with $0.1 \%$ PBST, rhodamineconjugated donkey anti-mouse anti-serum (1:100; Jackson ImmunoResearch, West Grove, PA) was used as secondary antibody. Hoechst staining (1:100; Sigma) was used for localization of nuclei and labeled cells were embedded in Mounting medium (Dako) before fluorescence microscopy (Zeiss).

Flow cytometry. Fluorescence-activated cell sorting (FACS) was performed on 4 stomach adenocarcinoma cell lines to quantify the expression levels of CD97. Cells $\left(2 \times 10^{5}\right)$ were resuspended in ice-cold PBS (Applichem, Darmstadt, Germany) with $10 \%$ FCS and $1 \%$ sodium azide, then incubated at room temperature for $30 \mathrm{~min}$ with primary antibody (CD97, 1:5,000, Abnova). After 3 times of washing, the cells were incubated with rhodamine-conjugated donkey anti-mouse anti-serum (1:100; Jackson ImmunoResearch, West Grove, $\mathrm{PA})$ at $4^{\circ} \mathrm{C}$ for $30 \mathrm{~min}$. FACS data were acquired on a FACS Calibur (Becton-Dickinson, Heidelberg, Germany), fluorescence data of 10,000 events were recorded and analyzed using CELL Quest software (Becton-Dickinson).

Statistical analysis. Statistical analysis was performed with the SPSS 10.0 software (SPSS GmbH, Munich, Germany). Student's t-test and one-way analysis of variance were used. All experiments were performed at least in triplicates and were expressed as mean \pm SEM with P-values of $<0.05$ considered as statistically significant.
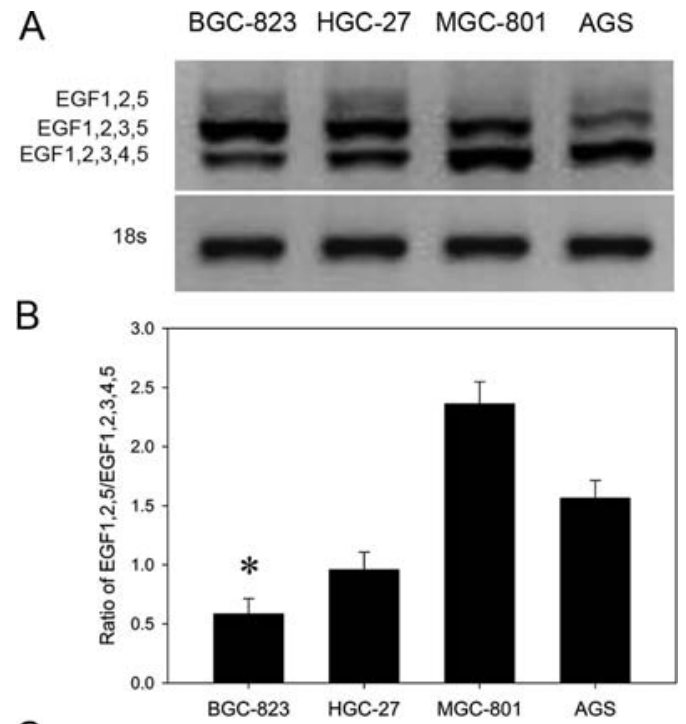

C
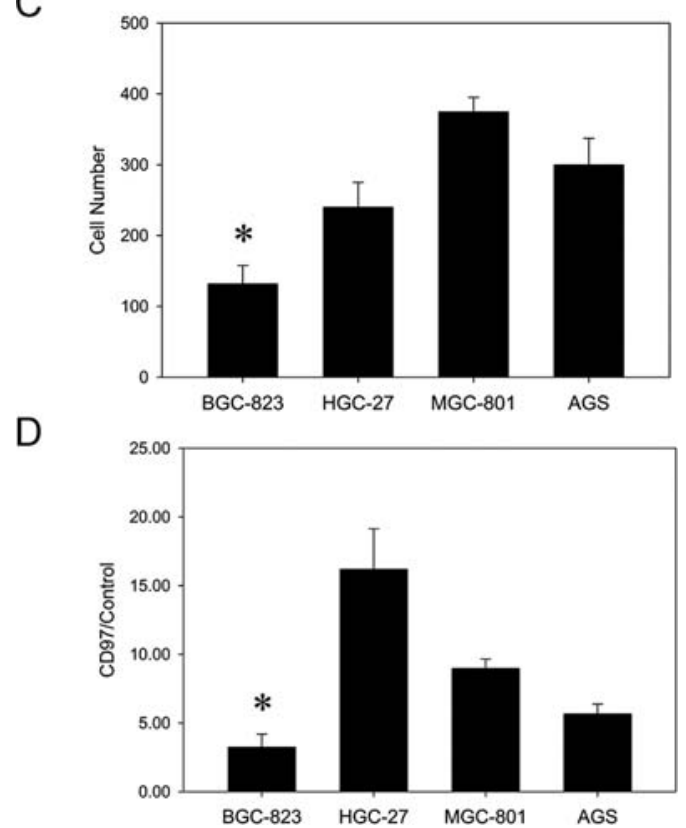

Figure 1. CD97 expression on stomach adenocarcinoma cell lines. RT-PCR (A) and densitometric (B) analysis performed on gastric cancer cell lines BGC-823, HGC-27, MGC-801 and AGS revealed that all cell lines investigated expressed mRNA of CD97 with various isoform distribution. 18S transcript levels were used as reference. Semi-quantitative RT-PCR evaluation was performed with the Bio 1D software. (C) Migration of the cells was performed on gelatin pre-coated Transwell membranes. The cells which traversed through the gelatin towards the lower surface of the membrane were stained after $24 \mathrm{~h}$ with $0.1 \%$ toluidine blue and counted by light microscopy. One-way ANOVA analysis was performed. The number of migrated BGC-823 cells was significantly lower $\left({ }^{*} \mathrm{p}<0.01\right)$ as compared with the other 3 cell lines. (D) FACS analysis revealed that BGC-823 cells had the lowest population of CD97-positive cells.

\section{Results}

Expression of CD97 in different gastric cancer cell lines. Four human gastric cancer cell lines, AGS, BGC-823, HGC-27 and MGC-801 were investigated for the CD97 gene and protein expression. RT-PCR revealed that all cell lines investigated expressed CD97, however with different isoform distribution (EGF1,2,5; EGF1,2,3,5 and EGF1,2,3,4,5). As demonstrated in Fig. 1A, BGC-823 cells had the lowest intensity of CD97/ 

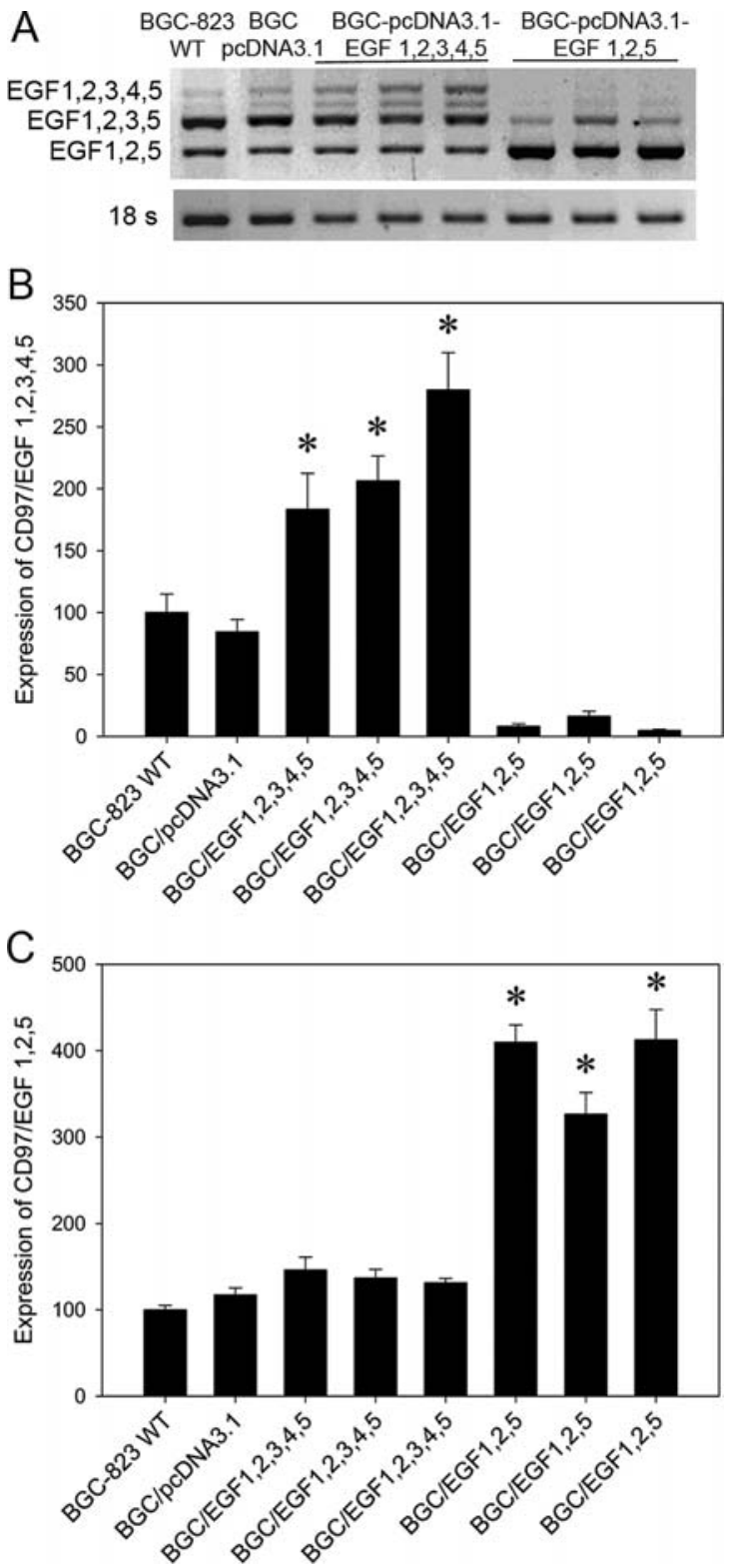

Figure 2. Expression of CD97 transcripts in stably transfected BGC-CD97 cells. (A) RT-PCR analysis was performed on CD97 full length isoform (856 bp) transfectants (CD97/EGF1,2,3,4,5), CD97 small isoform (577 bp) transfectants (CD97/EGF1,2,5), empty plasmid control (BGC/pcDNA3.1) and BGC-823 wild-type cells. $18 \mathrm{~S}$ transcript levels were used as reference. Semi-quantitative RT-PCR analysis, performed with the Bio 1D software, revealed significantly increased levels of (B) full length and (C) small CD97 isoforms as compared with corresponding controls $\left({ }^{*} \mathrm{p}<0.01\right)$.

EGF1,2,5 isoform, but the highest of CD97/EGF1,2,3,4,5. Furthermore, the ratio between EGF1,2,5 and EGF1,2,3,4,5 isoforms in BGC-823 cells was very low when comparing with MGC-801 and AGS cells, possessing much higher levels of CD97/EGF1,2,5 isoform and EGF1,2,5/EGF1,2,3,4,5 ratios (Fig. 1B). Investigations on protein level, by employing Western blotting, revealed much stronger CD97 expression in AGS, HGC-27 and MGC-801 comparing with nearly CD97 negative BGC-823 cells. The results of Western blotting were further verified by FACS analysis (Fig. 1D). In order to investigate the motile abilities of previously mentioned gastric cancer cells, we employed a migration assay. Out of

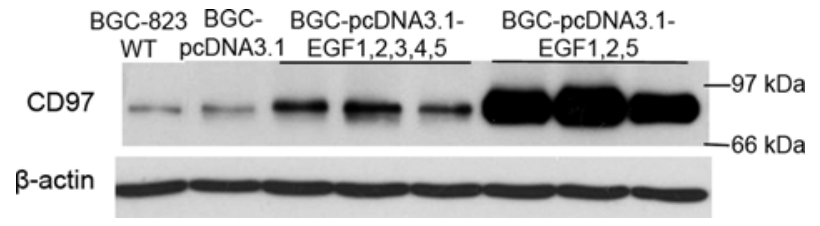

Figure 3. CD97 protein levels in stable BGC-CD97 transfectants. The $\sim 85 \mathrm{kDa}$ CD97 protein was detected by Western blot analysis in total cellular extracts of BGC-823 wild-type cells, BGC-CD97/EGF1,2,3,4,5, BGC-CD97/EGF1,2,5 and BGC/pcDNA3.1 transfectants. All BGC-CD97 transfectants expressed elevated levels of CD97 protein when compared with empty vector controls. $ß$-actin levels were used as reference.

four cell lines investigated, BGC-823 cells revealed the lowest number of migrated cells, which closely correlated with decreased CD97/EGF1,2,5 transcripts and EGF1,2,5/ EGF1,2,3,4,5 ratio, and reduced CD97 protein level (Fig. 1C).

Generation of gastric cancer cells with stable CD97 overexpression. BGC-823 cell line, which expressed low level of CD97 protein, was selected for stable transfection with pcDNA3.1-CD97/EGF1,2,3,4,5 or pcDNA3.1-CD97/EGF1,2,5 constructs. Over-expression of both CD97 isoforms was confirmed by RT-PCR in Fig. 2A. We noted that in CD97/ EGF1,2,5 clones, the up-regulation of CD97 small isoform (3.8 times higher) significantly coincided with the decrease of full length CD97 isoform (10.3 times lower). Reversely, in CD97/EGF1,2,3,4,5 clones, over-expression of CD97 full length isoform (2.2 times higher) did not significantly affect the expression of CD97 small isoform (Fig. 2B and C). Western blot analysis further confirmed the over-expression of CD97 in both clones comparing with wild-type cells or empty control. We detected a single specific immunoreactive band at $\sim 80 \mathrm{kDa}$ corresponding to CD97, which was very weak in empty plasmid clones and wild-type cells, but much stronger in CD97 transfectants. Quantification of CD97 expression in transfectants revealed that the intensity of overexpressed small CD97 isoform was $\sim 75$-fold higher than that of CD97 full length isoform (Fig. 3). Immunocytochemistry identified the membrane located CD97 expression, moderately up-regulated in BGC-CD97/EGF1,2,3,4,5 and highly elevated in BGC-CD97/EGF1,2,5 clones as compared with corresponding controls (Figs. 4 and 5).

CD97 isoforms play different roles in migration and invasion of human gastric cancer cells. Soft agar colony formation assay, performed on BGC-CD97/EGF1,2,3,4,5 or BGC-CD97/ EGF1,2,5 transfectants revealed their quite different invasive abilities as compared with empty plasmid cells. BGC-CD97/ EGF1,2,5 transfectants generated 5 times more colonies as compared with BGC-CD97/EGF1,2,3,4,5 clones and 2 times more than controls (Fig. 6A-C). Furthermore, the colonies formed by BGC-CD97/EGF1,2,5 transfectants were smaller and more disseminated. Contrary, BGC-CD97/EGF1,2,3,4,5 transfectants created much less and bigger-sized colonies as compared with control cells (Fig. 6D). To investigate whether CD97 may affect the extracellular matrix by alterations of penetrating ability, BGC-823 wild-type cells or CD97 transfectants were seeded on filters coated with gelatin. 


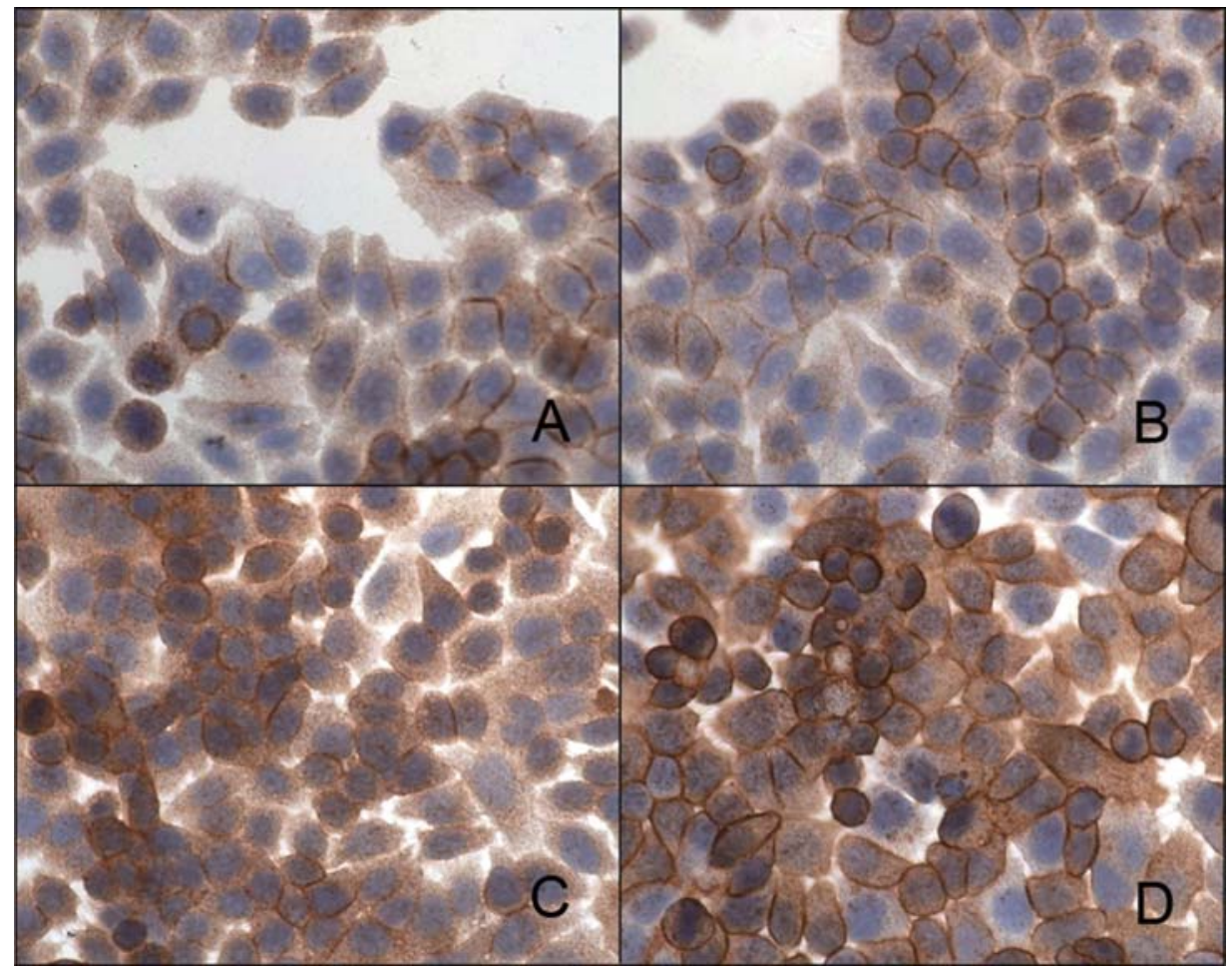

Figure 4. CD97 immunolocalization of BGC-CD97 transfectants. (A) BGC-823 wild-type cells; (B) BGC/pcDNA3.1 emtpy plasmid transfectants; (C) BGCCD97/EGF1,2,3,4,5 transfectants; (D) BGC-CD97/EGF1,2,5 transfectants. CD97 expression is mainly localized on the cell membrane, it is moderately elevated in BGC-CD97/EGF1,2,3,4,5 transfectants, but highly elevated in BGC-CD97/EGF1,2,5 transfectants.
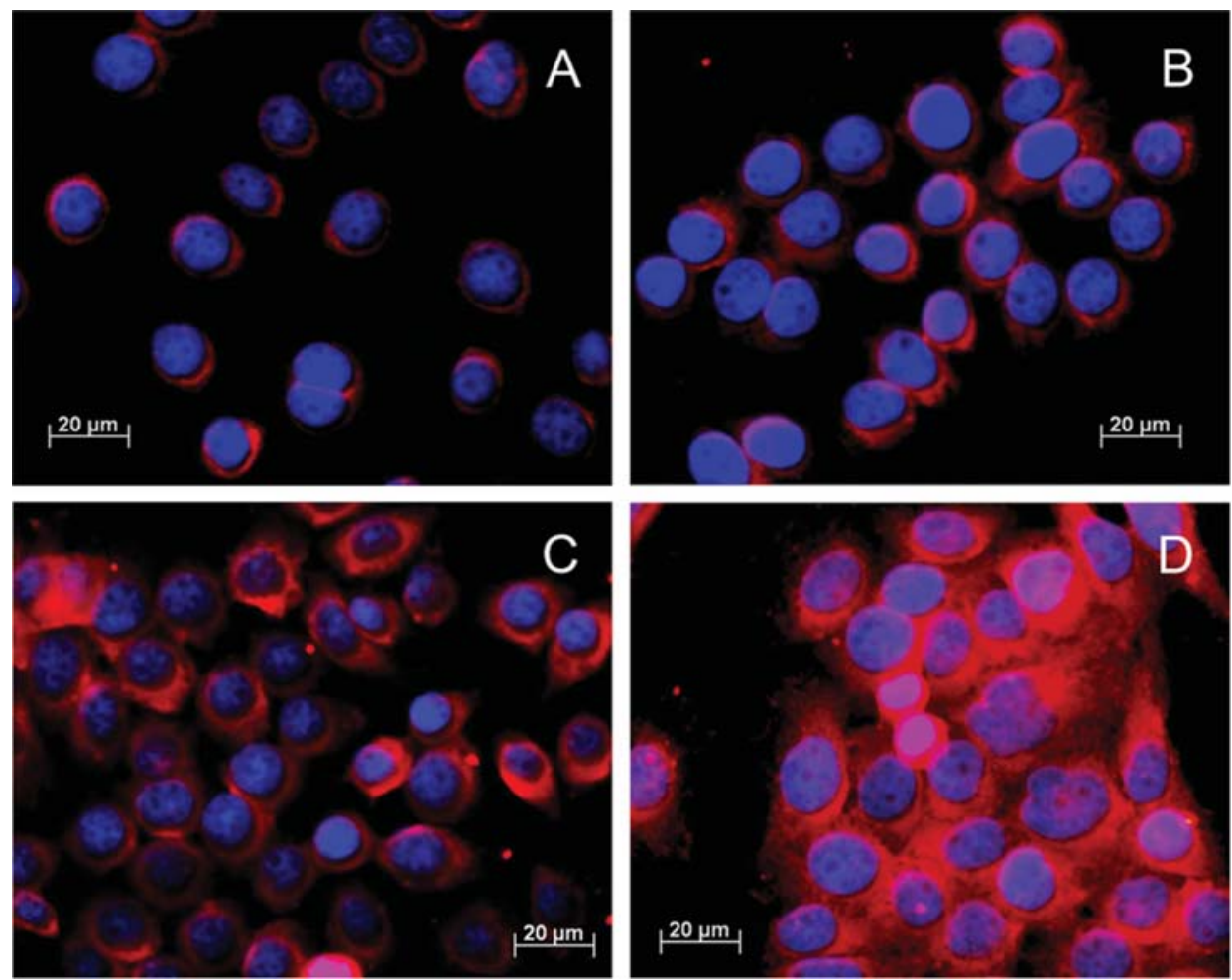

Figure 5. CD97 immunofluorescence of BGC-CD97 transfectants. (A) BGC-823 wild-type cells; (B) BGC/pcDNA3.1 transfectants; (C) BGC-CD97/ EGF1,2,3,4,5 transfectants; (D) BGC-CD97/EGF1,2,5 transfectants. CD97 expression, mainly localized on the cell membrane, is highly elevated in BGCCD97/EGF1,2,5 transfectants.

After 24-h incubation in the upper chamber, the cells that had migrated to the other side of the filter were counted. The number of penetrated CD97 small isoform clones was significantly increased when compared with empty plasmid controls and 2 times higher than the CD97 full length isoform clones (Fig. 6E-H). 


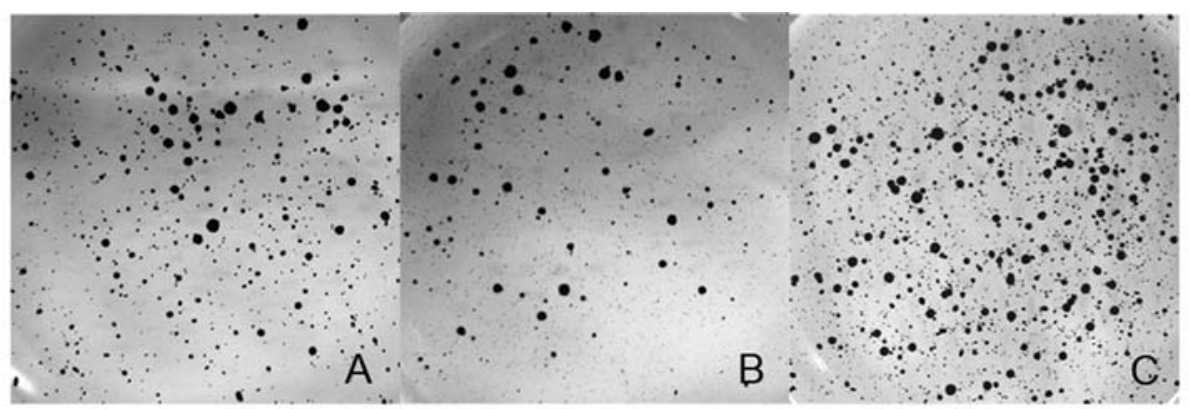

D
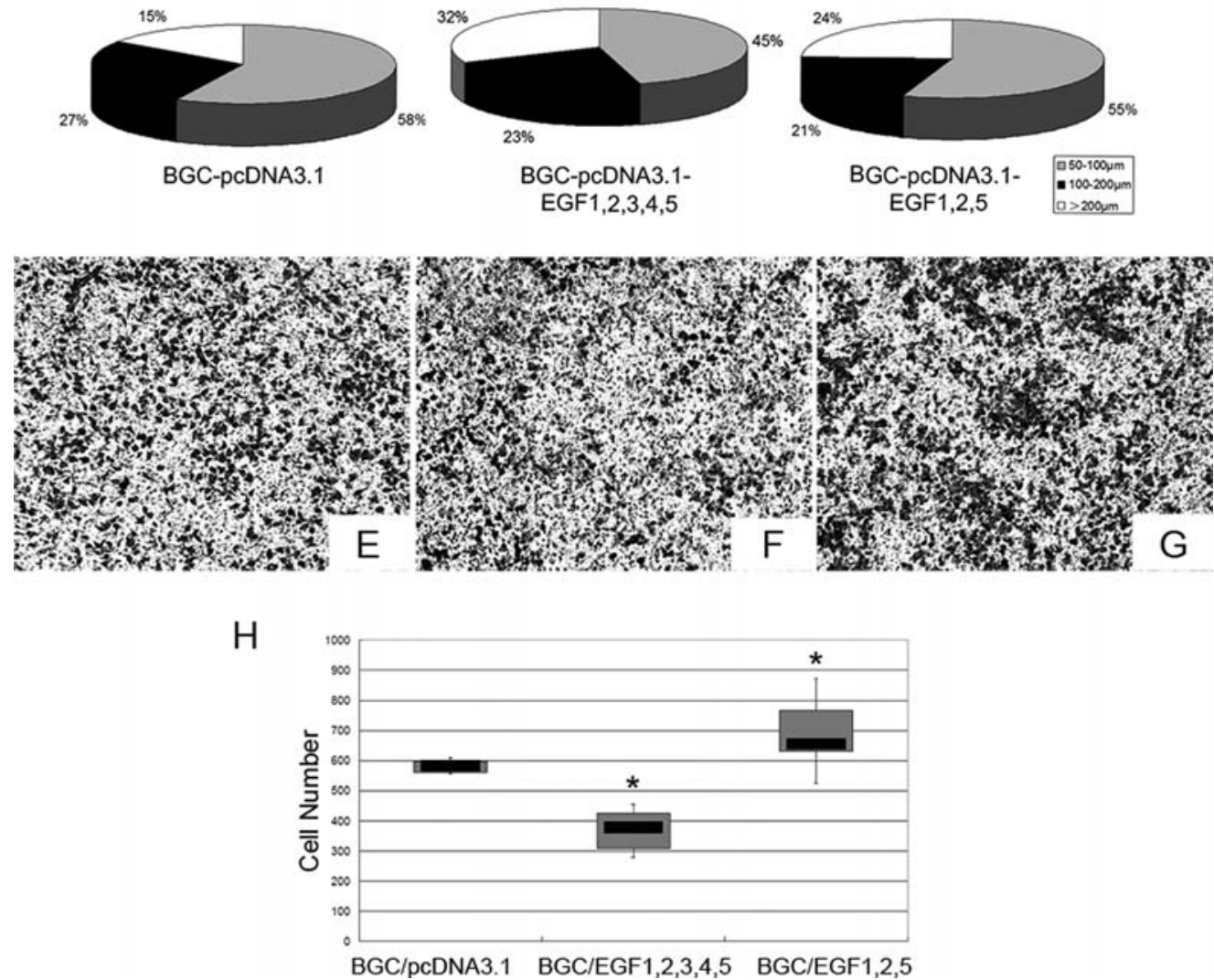

Figure 6. Soft agar colony assay and invasion assay. Invasion abilities of (A and E) BGC/pcDNA3.1 (empty control); (B and F) BGC-CD97/EGF1,2,3,4,5 and (C and G) BGC-CD97/EGF1,2,5 transfectants were tested by employing soft agar and migration assays. (D) The proportion of bigger, less invasive colonies $>200 \mu \mathrm{m}$ formed by BGC-CD97/EGF1,2,3,4,5 (32\%) was higher than those observed by BGC-CD97/EGF1,2,5 transfectants (24\%) and empty plasmid controls $(15 \%)$. Small and more invasive colonies $(50-100 \mu \mathrm{m})$ were more abundantly created by BGC/pcDNA3.1 and BGC-CD97/EGF1,2,5 cells as compared with the full length isoform. The number of cell colonies is presented as means of the counting results from 5 different areas. $(\mathrm{H})$ For the migration assay $2 \times 10^{4}$ of the cells were seeded on gelatin pre-coated membrane. After 24-h incubation, the cells which traversed to the lower surface of the membrane, were stained with $0.1 \%$ toluidine blue and counted by light microscopy. Experiments were performed in triplicates and five microscopic fields were counted for each filter. The results are shown in the graph as boxplot and one-way ANOVA analysis was performed. ${ }^{*}$-values $<0.01$ were considered as statistically significant.

Tubulin acetylation is one of the possible mechanisms affecting the invasiveness of CD97 transfected cells. In order to answer the question whether CD97-mediated changes in invasive ability are due to the alternation of cytoskeleton dynamics, CD97 transfectants, empty plasmid and wild-type cells were incubated with antibodies specific for $\alpha$-tubulin and acetylated tubulin. Several other tubulin modifications, such as tyrosination and polyglutamination, were also taken into account; however, no noticeable differences were demonstrated (data not shown). Immunofluorescence investigations revealed the changes in the proportion of $\alpha$-tubulin and acetylated tubulin within the CD97 transfectants. In our present study, a higher ratio of $\alpha$-tubulin/acetylated tubulin was detected in CD97 small isoform transfectants as compared with the full length isoform and corresponding control (Fig. 7). Tubulin acetylation status correlated well with increased motile and invasive abilities of BGC-CD97/ EGF1,2,5 cells and reduced aggressive-ness of BGC-CD97/ EGF1,2,3,4,5 clones.

\section{Discussion}

We demonstrated in our study the dual roles of CD97 isoforms in tumor cell migration and invasion. We found that the small 

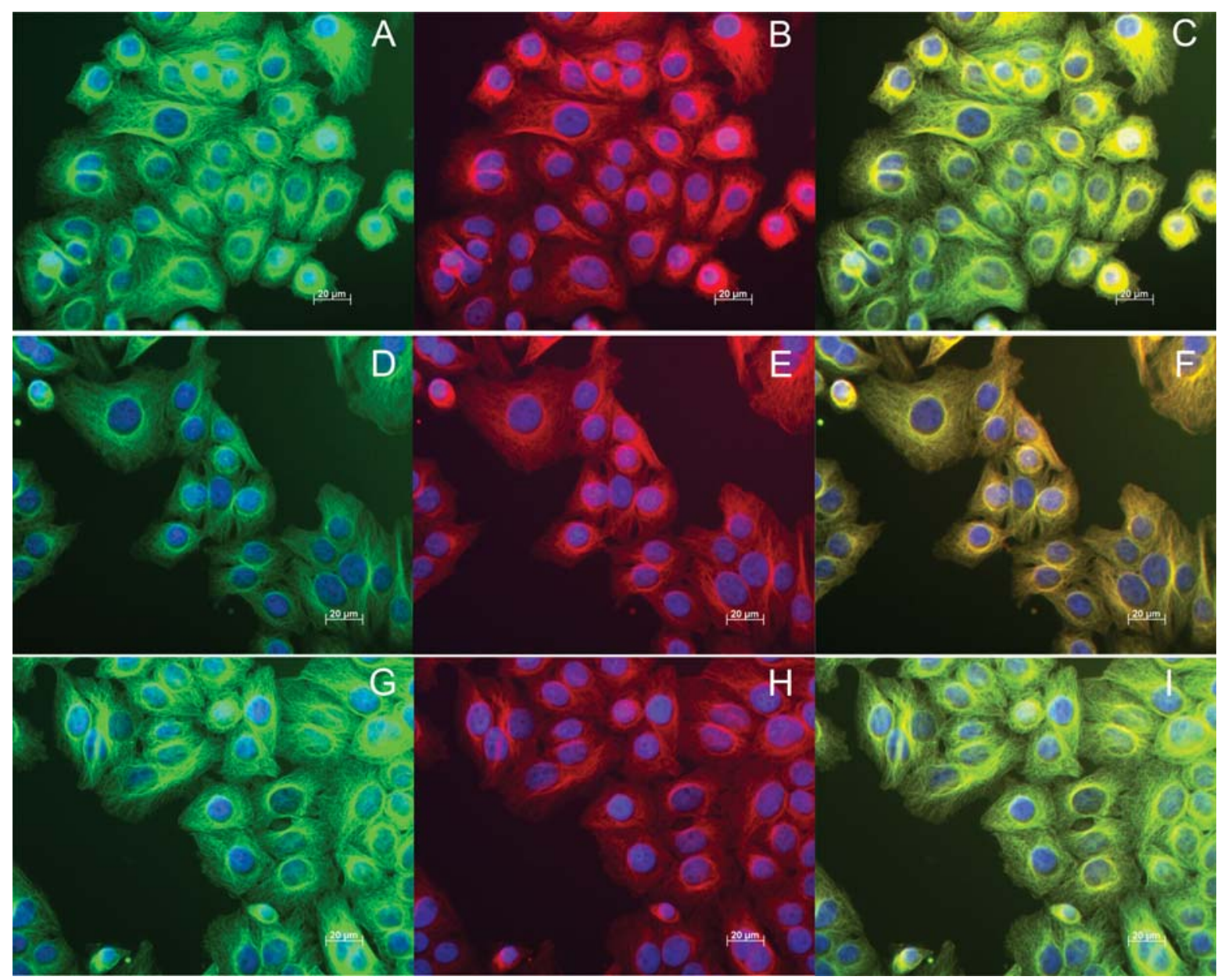

Figure 7. Immunofluorescence of $\alpha$-tubulin and acetylated tubulin. After fixation, permeabilization and blocking of non-specific binding, BGC/pcDNA3.1 (A-C), BGC-CD97/EGF1,2,3,4,5 (D-F) and BGC-CD97/EGF1,2,5 (G-I) clones were incubated overnight at $4^{\circ} \mathrm{C}$ with primary antibodies raised against acetylated-tubulin (red; B, E and H) and FITC-conjugated $\alpha$-tubulin (green; A, D and G); rhodamine conjugated donkey anti-mouse anti-serum was used as secondary antibody. $\mathrm{C}, \mathrm{F}$ and I are merged images of each clone, which revealed the lower ratio of $\alpha$-tubulin/acetylated-tubulin of BGC-CD97/EGF1,2,3,4,5 transfectants (F) as compared with BGC-CD97/EGF1,2,5 (I) and empty plasmid control (C).

CD97 isoform is linked with increased invasive behavior of gastric carcinoma cell line BGC-823, while the full length CD97 is associated with the tumor suppressive properties of these cells.

Various studies demonstrated overexpression of CD97 in tumor tissues, especially in scattered tumor cells at the tumor invasion front, possessing much higher motile abilities than the tumor cells in the solid formation $(18,19)$. Our previous study revealed that the strong CD97 expression as well as its ligand CD55 was exclusively localized at the tumor invasion front of gastric cancer, indicating participation of CD97 in the tumor cell migration and invasion (14). Also recent studies performed on gastric carcinoma tissues demonstrated a direct CD97 correlation with TNM status, however no relations with other clinicopathological parameters such as lymph node metastasis and the lymphoid/blood vessel infiltration were found (15). These observations suggest that overall CD97 protein expression may not possess a solitary correlation with the invasiveness of gastric carcinoma cells. Out of four gastric cancer cell lines investigated in this study, BGC-823 possessing the lowest total CD97 production, revealed noticeably downregulated transcripts of CD97 small isoform and decreased ratio of EGF1,2,5/EGF1,2,3,4,5, which further correlated with significantly reduced number of migrated cells. Previous investigations of three CD97 constructs, including small and full length isoforms, and C-terminal region, revealed that overexpression of CD97/EGF1,2,5 insert propagated invasion of colon cancer cells, while C-terminal insertion reduced it. The motility of the cells remained almost unaffected when the full length isoform was overexpressed (16). Based on these observations and our previous findings, we focused further investigations on the specific roles of CD97 isoforms in gastric carcinoma progression.

We demonstrated that $\mathrm{BGC}-823$ cells producing very low levels of endogenous CD97 protein were a suitable model to investigate the specific effects of CD97 isoforms. Over-expression of small CD97 isoform led to increased invasive capability as compared with the full length isoform and control cells. In contrast, introduction of the full length isoform led not only to the reduced motility of the cells, but also induced the impaired proteolytic activity, as demonstrated by our gelatin migration experiments. Furthermore, studies investigating the gelatinolytic abilities of CD97 transfected cells revealed that TNF- $\alpha$ stimulated fibrosarcoma 
cells HT1080, overexpresing small CD97 isoform, possessed the highest activity of matrix metalloproteinases (MMPs) MMP-2 and MMP-9 when compared with unstimulated wild-type or empty plasmid cells. HT1080 cells overexpressing the full length isoform of CD97 responded with almost unaltered or even decreased levels of MMPs when compared with corresponding controls (16). Considering these results, we assume that CD97/EGF1,2,3,4,5 isoform could be considered as a tumor suppressor comparing to invasion-promoting role of the CD97/EGF1,2,5 isoform. We suggest that the intrinsic imbalance between specific CD97 isoforms rather than the overall expression of the CD97 protein may affect the tumor cell behavior. This may further support our hypothesis, that distinct invasive abilities of other cell lines investigated in our study are specific CD97 isoforms dependent and coincide with the ratio of EGF1,2,5/ EGF1,2,3,4,5 isoforms, but not with the total CD97 expression.

We demonstrated that the higher proportion of $\alpha$-tubulin/ acetylated $\alpha$-tubulin correlated with the increased invasiveness of the CD97 small isoform clones, comparing with the full length or empty plasmid cells with decreased ratios of those proportions. It is well documented that increased acetylation of $\alpha$-tubulin is related to the stabilization and decreased dynamics of microtubules and the effect leads to the reduced motility of the cells (20-22). Although we can not exclude participation of other mechanisms, our findings suggest that CD97 isoforms may affect the microtubule dynamics, especially small isoform by preventing the acetylation of $\alpha$-tubulin and promoting the invasiveness of gastric carcinoma cells.

In conclusion, we demonstrated that the invasive abilities of gastric carcinoma cells are not only correlated with the total CD97 protein but also with the specific expression of CD97 isoforms. The CD97 small isoform may act as a tumor promoting agent, while the CD97 full length isoform as a tumor suppressor. Additionally, the maintenance of the balance between both isoforms may have an influence on the progression of gastric carcinoma. Down-regulation of the small CD97 isoform and subsequent promoting of full length isoform may have major implications in therapeutic strategies in the future.

\section{Acknowledgments}

This study was partly supported by the Science and Technology Bureau of Zhejiang Province, China (no. 2044C34010).

\section{References}

1. Martens GJ: Molecular biology of G-protein-coupled receptors. Prog Brain Res 92: 201-214, 1992.

2. Eichler W, Aust G and Hamann D: Characterization of an early activation-dependent antigen on lymphocytes defined by the monoclonal antibody BL-Ac(F2). Scand J Immunol 39: 111-115, 1994.

3. Chang GW, Stacey M, Kwakkenbos MJ, Hamann J, Gordon S and Lin HH: Proteolytic cleavage of the EMR2 receptor requires both the extracellular stalk and the GPS motif. FEBS Lett 547: $145-150,2003$.

4. Gray JX, Haino M, Roth MJ, Maguire JE, Jensen PN, Yarme A, Stetler-Stevenson MA, Siebenlist U and Kelly K: CD97 is a processed, seven-transmembrane, heterodimeric receptor associated with inflammation. J Immunol 157: 5438-5447, 1996.
5. Leemans JC, te Velde AA, Florquin S, Bennink RJ, De Bruin K, van Lier RA, van der Poll T and Hamann J: The epidermal growth factor-seven transmembrane (EGF-TM7) receptor CD97 is required for neutrophil migration and host defense. J Immunol 172: 1125-1131, 2004

6. Kwakkenbos MJ, Matmati M, Madsen O, Pouwels W, Wang Y, Bontrop RE, Heidt PJ, Hoek RM and Hamann J: An unusual mode of concerted evolution of the EGF-TM7 receptor chimera EMR2. FASEB J 20: 2582-2584, 2006.

7. Steinert M, Wobus M, Boltze C, Schütz A, Wahlbuhl M, Hamann J and Aust G: Expression and regulation of CD97 in colorectal carcinoma cell lines and tumor tissues. Am J Pathol 161: 1657-1667, 2002

8. Hamann J, Stortelers C, Kiss-Toth E, Vogel B, Eichler W and van Lier RA: Characterization of the CD55 (DAF)-binding site on the seven-span transmembrane receptor CD97. Eur J Immunol 28: 1701-1707, 1998.

9. Lin HH, Stacey M, Saxby C, Knott V, Chaudhry Y, Evans D, Gordon S, McKnight AJ, Handford P and Lea S: Molecular analysis of the epidermal growth factor-like short consensus repeat domain-mediated protein-protein interactions: dissection of the CD97-CD55 complex. J Biol Chem 276: 24160-24169, 2001.

10. Hoang-Vu C, Bull K, Schwarz I, Krause G, Schmutzler C, Aust G, Köhrle J and Dralle H: Regulation of CD97 protein in thyroid carcinoma. J Clin Endocrinol Metab 84: 1104-1109, 1999.

11. Jaspars LH, Vos W, Aust G, van Lier RA and Hamann J: Tissue distribution of the human CD97 EGF-TM7 receptor. Tissue Antigens 57: 325-331, 2001

12. Aust G, Eichler W, Laue S, Lehmann I, Heldin NE, Lotz O, Scherbaum WA, Dralle H and Hoang-Vu C: CD97: a dedifferentiation marker in human thyroid carcinomas. Cancer Res 57: 1798-1806, 1997.

13. Inoue T, Yamakawa M and Takahashi T: Expression of complement regulating factors in gastric cancer cells. Mol Pathol 55: 193-199, 2002.

14. Liu Y, Chen L, Peng SY, Chen ZX and Hoang-Vu C: Role of CD97(stalk) and CD55 as molecular markers for prognosis and therapy of gastric carcinoma patients. J Zhejiang Univ Sci B 6: 913-918, 2005.

15. Aust G, Steinert M, Schütz A, Boltze C, Wahlbuhl M, Hamann J and Wobus M: CD97, but not its closely related EGF-TM7 family member EMR2, is expressed on gastric, pancreatic, and esophageal carcinomas. Am J Clin Pathol 118: 699-707, 2002.

16. Galle J, Sittig D, Hanisch I, Wobus M, Wandel E, Loeffler M and Aust G: Individual cell-based models of tumor-environment interactions: multiple effects of CD97 on tumor invasion. Am J Pathol 169: 1802-1811, 2006.

17. Hensel F, Hermann R, Brändlein S, Krenn V, Schmausser B, Geis S, Müller-Hermelink HK and Vollmers HP: Regulation of the new coexpressed CD55 (decay-accelerating factor) receptor on stomach carcinoma cells involved in antibody SC-1-induced apoptosis. Lab Invest 81: 1553-1563, 2001.

18. Park KJ, Choi HJ, Roh MS, Kwon HC and Kim C: Intensity of tumor budding and its prognostic implications in invasive colon carcinoma. Dis Colon Rectum 48: 1597-1602, 2005.

19. Nakanishi Y, Ochiai A, Kato H, Tachimori Y, Igaki H and Hirohashi S: Clinicopathological significance of tumor nest configuration in patients with esophageal squamous cell carcinoma. Cancer 91: 1114-1120, 2001.

20. Maruta H, Greer K and Rosenbaum JL: The acetylation of alpha-tubulin and its relationship to the assembly and disassembly of microtubules. J Cell Biol 103: 571-579, 1986.

21. Honore S, Kamath K, Braguer D, Wilson L, Briand C and Jordan MA: Suppression of microtubule dynamics by discodermolide by a novel mechanism is associated with mitotic arrest and inhibition of tumor cell proliferation. Mol Cancer Ther 2: 1303-1311, 2003.

22. Westermann S and Weber K: Post-translational modifications regulate microtubule function. Nat Rev Mol Cell Biol 4: 938-947, 2003. 